\title{
Two minute slope inhalation technique for cerebral blood flow measurement in man
}

\author{
1. Method \\ DAVID J. WYPER, GORDON A. LENNOX, AND JOHN O. ROWAN \\ From the M.R.C. Cerebral Circulation Research Group, Institute of Neurological Sciences, and \\ Department of Clinical Physics and Bioengineering, Glasgow
}

SYNOPSIS The two minute slope method of measuring cerebral blood flow (CBF) by inhalation of ${ }^{133} \mathrm{Xe}$ is described. It requires the subject to remain still for only two minutes and is thus applicable to a wide variety of patients and to investigations involving brief physiological stimuli. The influence of extracerebral blood flow on CBF calculated by this method has been studied. Results from the two minute slope inhalation method using two minutes of data correlate well with those obtained by carotid injection using either two minutes or 10 minutes of data.

Quantitative measurements of cerebral blood flow (CBF) in man have been possible for more than 20 years, using the inert gas clearance method. In the early years nitrous oxide inhalation was used (Kety and Schmidt, 1945, 1948) with sampling of jugular venous concentrations, but in the last 12 years the most common method has been to measure the wash-out of radioactive tracers using external detectors placed over the skull (Glass and Harper, 1963). It has so far been held that the only reliable way to introduce the isotope is by injection into the carotid artery in the neck, and this has limited the application of CBF measurements in clinical practice largely to patients already undergoing carotid angiography. For this reason, considerable attention is now being paid to atraumatic techniques, and in particular the ${ }^{133} \mathrm{Xe}$ inhalation technique. This gives rise to two problems which do not apply with intracarotid injection of ${ }^{133} \mathrm{Xe}$ : recirculation of isotope from the rest of the body, and contamination of extracerebral tissue.

There is a variety of versions of the inhalation technique using analogue computing (Mallett and Veall, 1965; Crawley, 1968) or digital computing (Obrist et al., 1967; Kral et al., 1973), with inhalation times varying from one minute to five minutes, and measurement times ranging from two minutes to 50 minutes.

(Accepted 9 September 1975.)
The method described here, called the two minute slope method, has been developed in order to reduce the duration of each investigation so as to minimise changes in other physiological variables during the course of CBF measurement and to make it practical for patients with limited tolerance or cooperation.

\section{METHODS}

PROCEDURE The respiratory apparatus used (Fig. 1) consists of a 61 spirometer with three-way taps at the inlet and outlet to permit switching between open and closed loop systems. A mixture of ${ }^{133} \mathrm{Xe}$ in air is introduced into the spirometer from a pressurized cylinder immediately before the investigation to produce a specific activity of approximately $\mathbf{1 . 0}$ $\mathrm{mCi} /$ litre.

The mouthpiece normally used is of the rubber insert type, but, for the occasional patient who encounters difficulty with this, an RAF pilot's mask can be used. A fine polyethylene tube is taken from the mouthpiece through an infra-red $\mathrm{CO}_{2}$ analyser (Capnograph) to measure the end-tidal $\mathrm{PCO}_{2}$ $\left(\mathrm{P}_{\mathrm{et}} \mathrm{CO}_{2}\right)$ and then through a well-type scintillation counter to measure the concentration of ${ }^{133} \mathrm{Xe}$ in the end-expired air. Adequate counting statistics are obtained in the well counter by winding the tube in a spiral inside the well. Analogue outputs from the $\mathrm{CO}_{2}$ analyser and scintillation counter/ratemeter system are written out on a two-channel chart recorder 141 


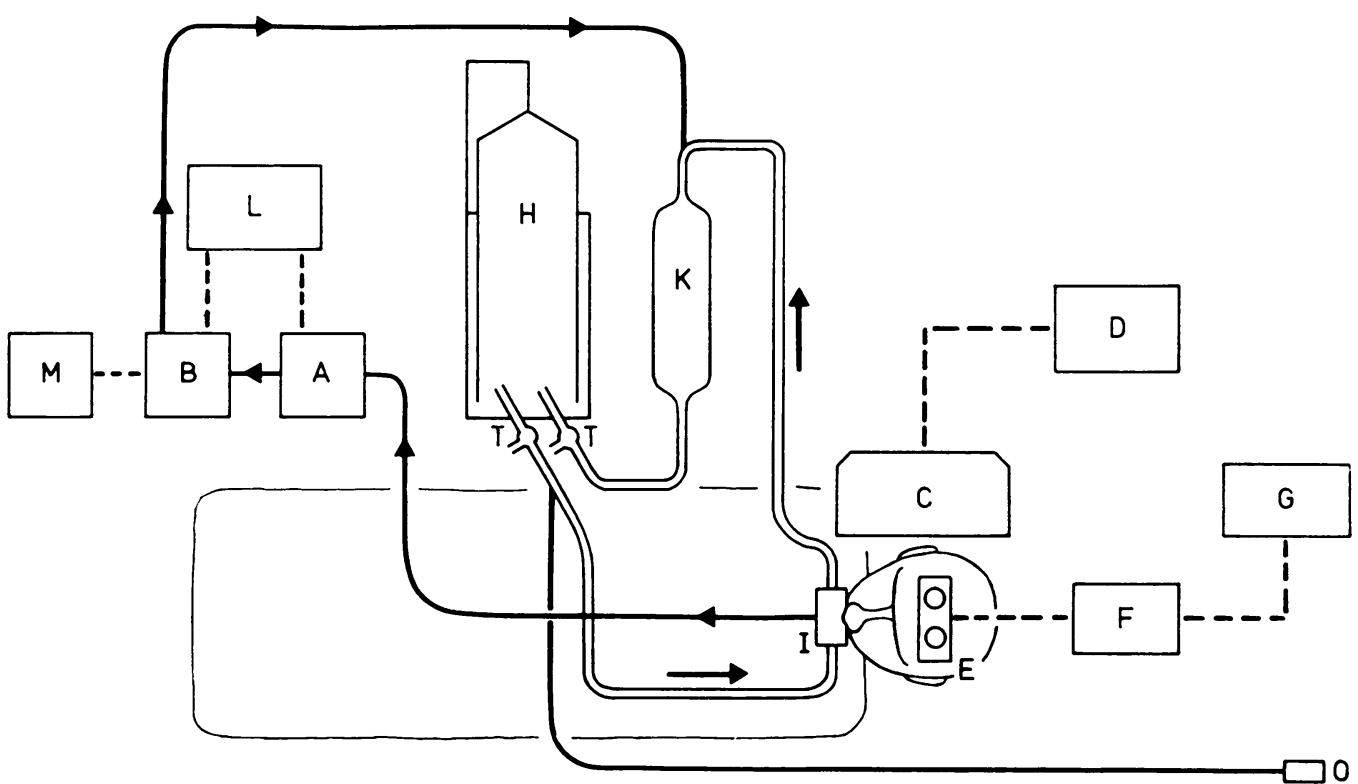

FIG. 1 Diagram of $\mathrm{CBF}$ measurement system. A-CO analyser. B-Well scintillation counter. $\mathrm{C}-$ Gamma $\overrightarrow{0}+\overrightarrow{\mathrm{O}}$ camera. D-Gamma camera console. E-Dual detector heads. F-Dual detector nucleonics. G-Teletype $\overrightarrow{\mathbb{D}} \vec{\circ}$ $\mathrm{H}-$ Spirometer. I-Mouthpiece. $\mathrm{K}-\mathrm{CO}_{2}$ (soda lime) absorber. L-Servoscribe. $\mathrm{M}-$ Nucleonics for well detector. T-Three-way taps. O-Oxygen supply. - - Electrical connections. — Gas connections.

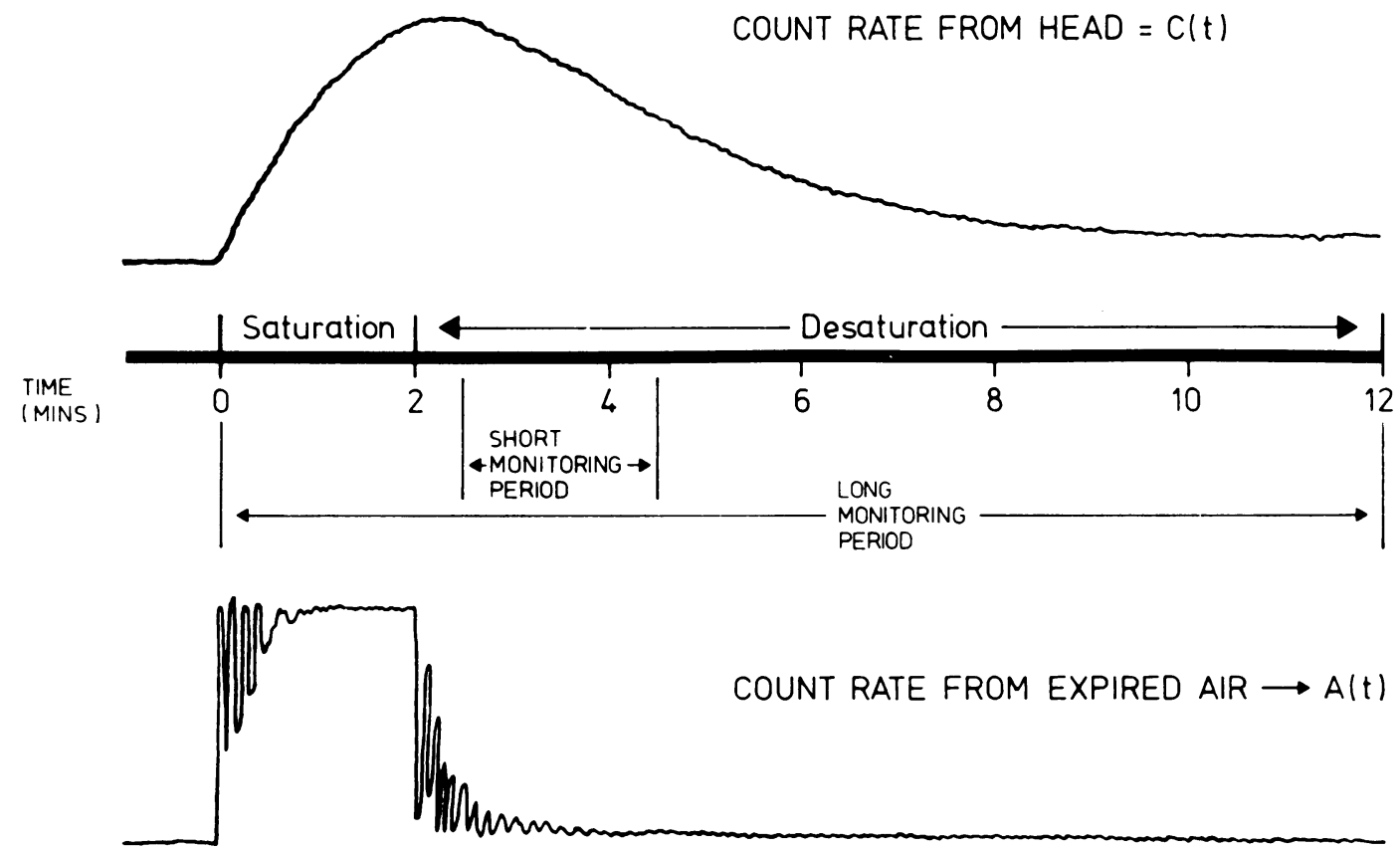

FIG. 2 Experimental protocol for two minute slope analysis or for a full 12 minute bicompartmental analysis. 
The head is viewed by either four collimated scintillation counters which have a resolution diameter of $4.0 \mathrm{~cm}$ at distance in tissue of $6.0 \mathrm{~cm}$ or by a gamma camera collimated to have a similar resolution. The subject is positioned in relation to the detector and is instructed about voluntary control of respiration. After an initial acclimatization period of breathing on the open loop system, and when the $\mathrm{P}_{\mathrm{et}} \mathrm{CO}_{2}$ is steady at the desired value, a background count is measured (Fig. 2). The taps are then turned to produce the closed loop breathing system and the ${ }^{133} \mathrm{Xe}$-air mixture is breathed for two minutes, at the end of which it is abruptly stopped by reverting to the open loop breathing system. To conform with the mathematical assumptions, 30 seconds are allowed to elapse before recording the clearance from the head for two minutes. The calculation of clearance rates from the head using the PDP-12 computer takes eight minutes if the gamma camera is used but only a few seconds with the single channel detectors.

THEORY An 'initial slope' index has been used for a number of years to calculate mean CBF after intracarotid injection of isotope (Paulson et al., 1969; Olesen et al., 1971). The assumption underlying this method is that, for the first one or two minutes after injection, the mean slope of the clearance curve, on a semi-logarithmic scale, is proportional to mean CBF. The results obtained using this method tend to reflect blood flow in the faster compartment of the brain.

Using a PDP-12 computer, we have synthesized clearance curves using various arterial concentration functions and a range of values of cerebral blood flow. When plotted on a semilogarithmic scale, these curves are approximately linear for a two minute period starting 30 seconds into the desaturation phase after inhalation. Clearance curves recorded from patients were similar to these synthetic curves. This indicated that an initial slope method of analysis would be at least as valid after inhalation as after injection.

The equation implicit in this assumption is

$$
\mathrm{c}(\mathrm{t})=\mathrm{Be}^{-\mathrm{K}_{\mathrm{H}} \mathrm{t}} 2.5<\mathrm{t}<4.5
$$

$\mathrm{K}_{\mathrm{H}}$ will be referred to as the clearance rate from the head.

The passage of tracer through the brain is represented by the equation (Kety, 1972):

$$
c(t)=K \lambda e^{-K t} \int_{0}^{t} A(\mu) e^{K \mu} d \mu
$$

where $\mathrm{t}=$ time after start of saturation phase

c = concentration in brain

A $=$ arterial concentration

$$
\begin{array}{ll}
\lambda \quad= & \text { partition coefficient between blood and } \\
& \text { brain } \\
\mathrm{K}=\mathrm{CBF} / \lambda & \\
\mathrm{CBF} & =\text { blood flow per unit weight of tissue } \\
\mu \quad=\mathrm{a} \text { 'dummy' time variable }
\end{array}
$$

As with the stochastic height/area technique (Zierler, 1965; Wyper and Rowan, 1972), mean flow is measured and not grey and white matter flows. The partition coefficient used is therefore somewhat arbitrary because the relative weights of grey and white matter perfused with ${ }^{133} \mathrm{Xe}$ depend on the flow in each compartment, the relative mass of each tissue, the inhalation time, and the part of the clearance curve over which flows are measured. We calculated a mean partition coefficient of 1.14 using mean grey and white matter flows and weights obtained using the ${ }^{133} \mathrm{Xe}$ injection technique on 19 patients with varying pathologies. This assumes a grey matter partition coefficient of 0.8 and a white matter partition coefficient of 1.5 (Lassen and Ingvar, 1972). Using the data of Wilkinson et al. (1969) on 10 normal subjects, a value of 1.10 was obtained for mean partition coefficient.

Equation (1) is used to compute $K_{H}$ for each clearance curve, a least squares linear regression being used. An iterative technique is then used to find $K$ in equation (2). The least squares regression programme is written in PDP-12 assembly language

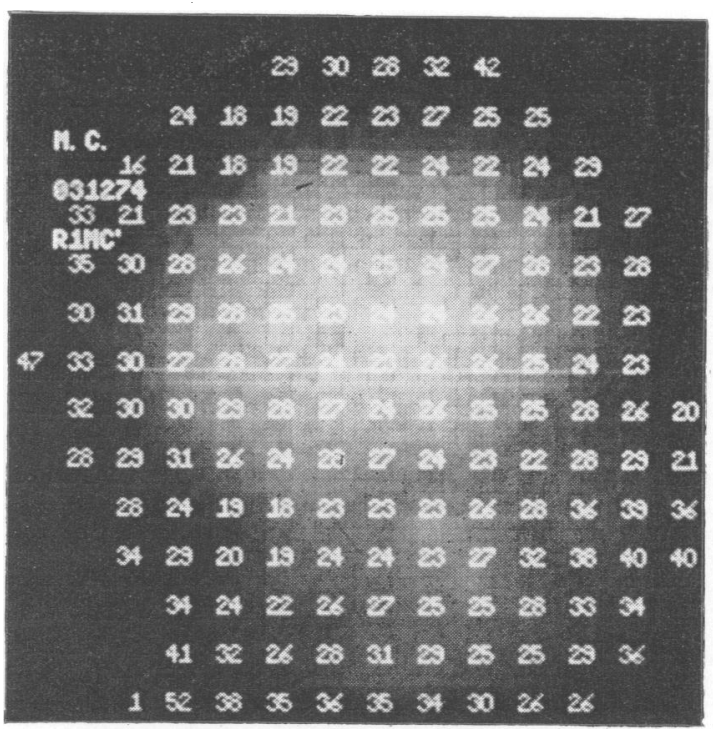

FIG. $3 A$ digital map of $K_{\mathrm{H}}$ values superimposed on a varying grey shade of the static distribution of ${ }^{133} \mathrm{Xe}$. This is a normal right lateral view. 
$\%$ Reduction

in Measured CBF

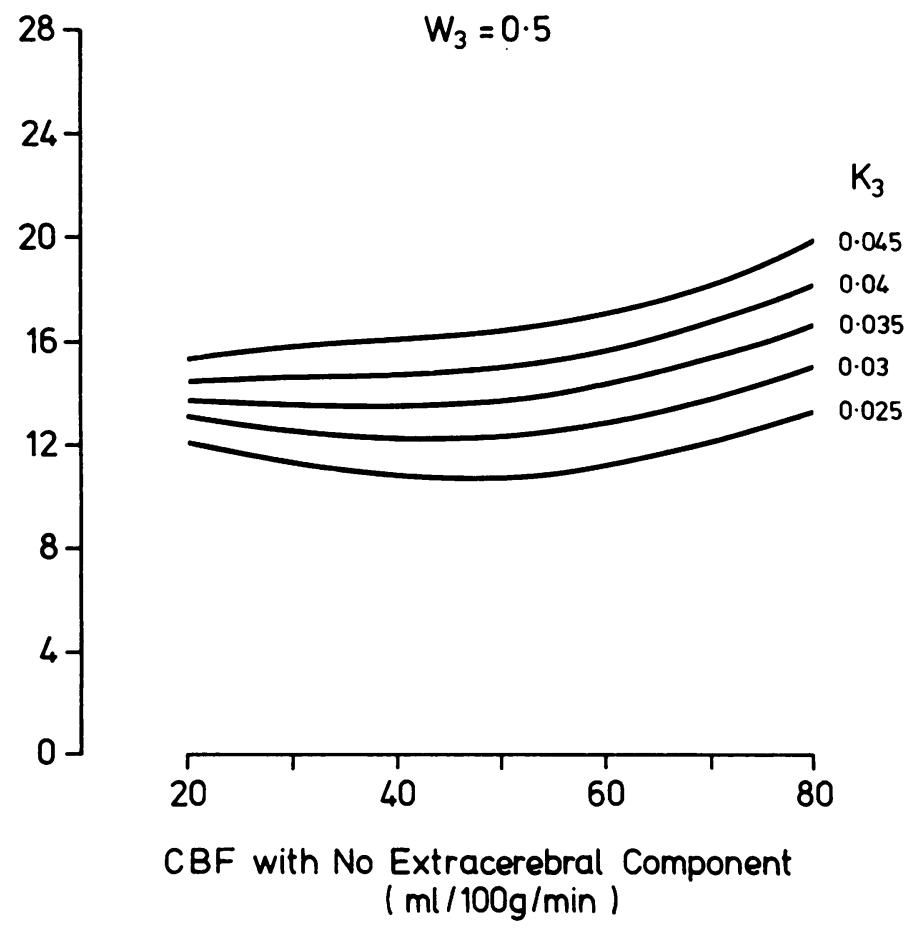

FIG. 4 The percentage reduction is measured $C B F$ due to the presence of a third compartment. $W_{3}$ is the relative weight of the third compartment $\left(W_{1}+W_{2}=1\right)$ and $K_{3}$ the clearance rate from this compartment.

and occupies approximately eight minutes of computing time for calculation of 256 values of $K_{H}$ but only a few seconds for a single value. Any flow may then be computed using the iterative technique, the programme for this being written in the PDP-12 high level language FOCAL-12. The run time for this is about one minute.

Production of flow maps If data are recorded using the gamma camera, then, for each element within the $16 \times 16$ matrix, $\mathrm{K}_{\mathrm{H}}$ is computed. The count rate per matrix element is about 50 counts per second in the region viewed by the brain. If the total integrated count in any set of corresponding matrix elements does not exceed a predetermined threshold level, the value of $K_{H}$ is set equal to zero, thereby eliminating areas outside the head. A digital map of $K_{H}$ values is displayed on the computer screen (Fig. 3). It is superimposed on a varying grey shade map of the static distribution of ${ }^{133} \mathrm{Xe}$ during one of the epochs of four seconds in order to correlate $K_{H}$ values with anatomical position. For any value of $K_{H}$ flow can be calculated as described above.

\section{RESULTS}

EFFECT OF EXTRACEREBRAL BLOOD FLOW A theoretical disadvantage in using an inhalation as opposed to an injection technique to measure $\mathrm{CBF}$ is that extracerebral tissue becomes contaminated with ${ }^{133} \mathrm{Xe}$. If the clearance curve is followed for a sufficiently long time-for example, 30 to 50 minutes-then this so-called third component can be measured and separated from at least the faster component of CBF. With our short analysis, however, this is not possible, but with a two minute inhalation time the loading of this third component is relatively small. It is, nevertheless, important to investigate the errors likely to be introduced by the third component.

We have studied this by generating simulated three compartment clearance curves on our PDP-12 and then using the two minute slope analysis method to calculate CBF from these curves. Figure 4 shows that, for a range of values 


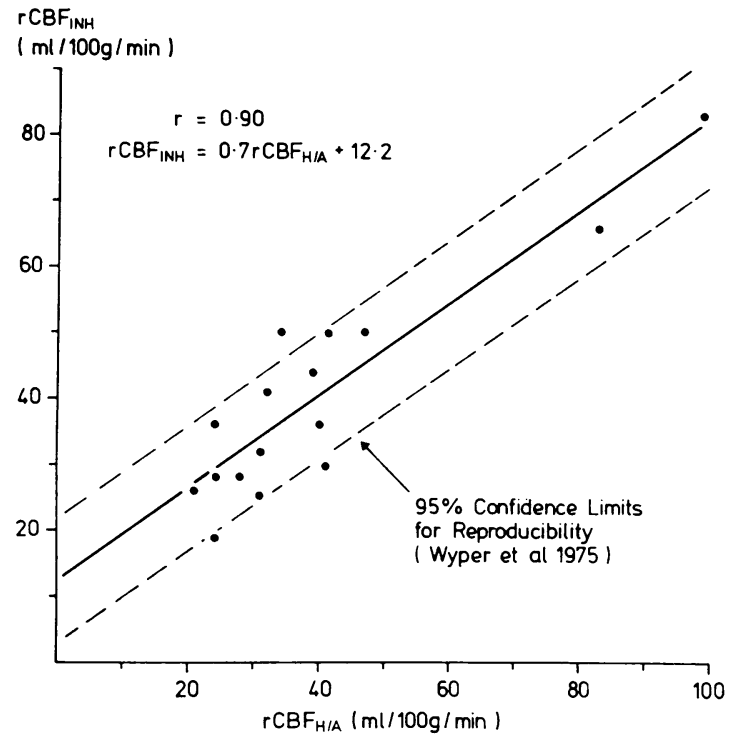

(a)

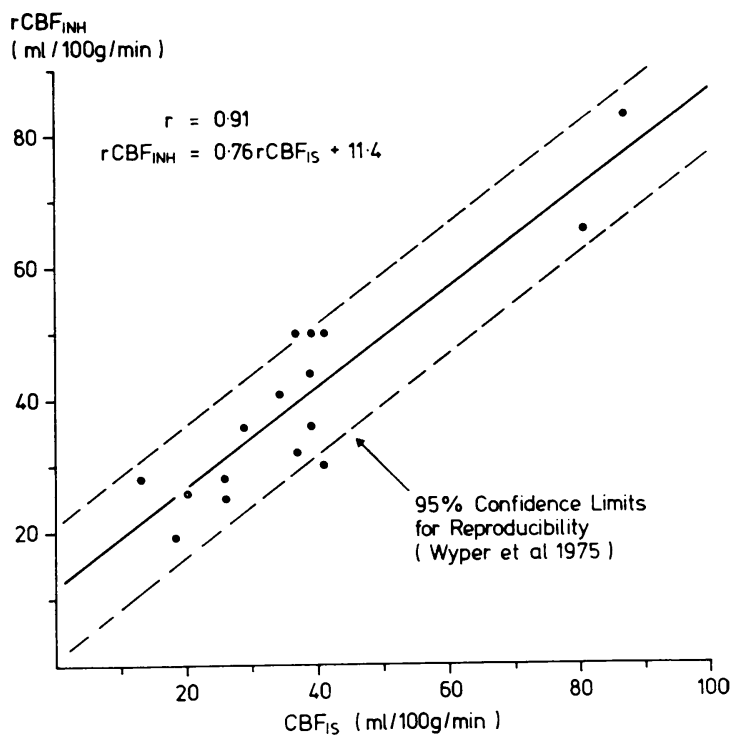

(b)

FIG. 5 The correlation between $r C B F_{\mathrm{INH}}$ and (a) $r C B F_{\mathrm{H} / \mathrm{A}}$ or (b) $r C B F_{\mathrm{I} . \mathrm{s}}$.

of clearance rate $\mathrm{K}_{3}$ greater than that normally encountered physiologically, the presence of the third component causes the value of CBF calculated to be lower by about $14 \%$ on average. This is relatively independent of CBF and of the flow of the third component.

INJECTION-INHALATION COMPARISON Results obtained from this technique have been compared with measurements made at the same session using the injection technique. After angiography, isotope was injected into the common carotid artery. The resulting clearance curves were analysed for mean flow using both the height over area (H/A) and initial slope (IS) methods

TABLE

CORRELATION COEFFICIENTS BETWEEN VARIOUS METHODS OF MEASURING CBF

\begin{tabular}{lcll}
\hline Two minute slope inhalation & vs & H/A injection & 0.90 \\
Two minute slope inhalation & vs & IS injection & 0.91 \\
Two minute slope inhalation & vs & WM injection & 0.91 \\
Two minute slope inhalation & vs & F injection & 0.87 \\
H/A injection & vs & WM injection & 0.995 \\
IS injection & vs & H/A injection & 0.96 \\
IS injection & vs & F injection & 0.96 \\
& & & \\
\hline
\end{tabular}

and were stripped biexponentially to obtain slow, fast (F) and weighted mean (WM) flows. After the 10 minute clearance from the injection of ${ }^{133} \mathrm{Xe}$, a measurement was made using inhalation-the ${ }^{133} \mathrm{Xe} /$ air mixture being added to the anaesthetic gases for a two minute period. In a few cases, where there were no clinical contraindications, a further two measurements were made after adjusting the ventilation to change $\mathrm{pCO}_{2}$. In all, 16 pairs of measurements were made in 11 subjects. The head was viewed laterally by two single channel scintillation counters, collimated to have a resolution of about $4.0 \mathrm{~cm}$.

Correlations between results (Fig. 5 and the Table) calculated by different methods are around 0.9 . More than $84 \%$ of the inhalation results fall within the $95 \%$ confidence limits for reproducibility when they are compared with the $\mathrm{H} / \mathrm{A}$ injection results and more than $89 \%$ if they are compared with the IS injection results.

Either $\mathrm{CBF}_{\mathrm{H} / \mathrm{A}}$ or $\mathrm{CBF}_{\mathrm{w} . \mathrm{M} \text {. }}$ is commonly accepted as standard for mean $\mathrm{CBF}$ and there is a close correlation between them $(r=0.995)$. Taking $\mathrm{CBF}_{\mathrm{H} / \mathrm{A}}$ as a standard, the correlation equation

$$
\mathrm{rCBF}_{\mathrm{INH}}=0.7 \mathrm{rCBF}_{\mathrm{H} / \mathrm{A}}+12.2
$$

transposes to:

$$
\mathrm{rCBF}_{\mathrm{H} / \mathrm{A}}=1.42\left(\mathrm{rCBF}_{\mathrm{INH}}-12.2\right)
$$


If it is wished to translate an inhalation result to an injection result this equation can be used.

\section{DISCUSSION}

The main advantage of the inhalation technique is that it is atraumatic and is therefore applicable to a much wider group of patients than is a method which depends on carotid puncture. It is also possible to use it for studies on normal subjects and both with them and with patients repeated measurements over hours, days, or weeks are possible. The radiation dose per measurement to the lungs (the critical organ) is $70 \mathrm{~m} . \mathrm{rad}$, which is less than half that from a chest radiograph.

The total time of the investigation using the two minute slope method is five minutes and the washout from the head is recorded for the final two minutes when the position of the head must be fixed in relation to the detectors. The use of such a brief time interval enables the patient to maintain reasonably steady state conditions, even if hypocapnic or hypercapnic responses are being studied. It has also enabled CBF measurements to be made on confused or demented patients. We also have the facility for a 12 minute bicompartmental analysis method but find we can use this in less than $20 \%$ of our investigations.

One difficulty encountered in practice is that of choosing a suitably fitting mouth piece and often several have to be tried.

CBF calculated by this method, which uses inhalation and two minutes of data, has been shown to correlate well with results using the H/A method, which uses intracarotid injection and 10 minutes of data.

We would like to thank Mr D. Keating for diligent technical assistance.

\section{REFERENCES}

Crawley, J. C. W. (1968). An analogue computer for calculating blood perfusion rates. Bio-medical Engineering, 3, 256-261.

Glass, H. I., and Harper, A. M. (1963). Measurement of regional cerebral blood flow in cerebral cortex of man through intact skull. British Medical Journal, 1, 593-595.

Kety, S. S. (1972). Study of the cerebral circulation by means of inert diffusible tracers. In Progress in Brain $R e$ search, vol. 35, pp. 375-385. Edited by Meyer and Schade, Elsevier: Amsterdam.

Kety, S. S., and Schmidt, C. F. (1945). The determination of cerebral blood flow in man by the use of nitrous oxide in low concentrations. American Journal of Physiology, 143, 53-66.

Kety, S. S., and Schmidt, C. F. (1948). The nitrous oxide method for the quantitative determination of cerebral blood flow in man: theory, procedure and normal values. Journal of Clinical Investigation, 27, 476-483.

Kral, M., Kuba, J., and Husak, V. (1973). Determination of regional cerebral blood flow using a ${ }^{133} \mathrm{Xe}$ inhalation technique. Physics in Medicine and Biology, 18, 100-107.

Lassen, N. A., and Ingvar, D. H. (1972). Radioisotopic assessment of regional cerebral blood flow. Progress in Nuclear Medicine, 1, 376-409.

Mallett, B. L., and Veall, N. (1965). The measurement off regional cerebral clearance rates in man using Xenon ${ }^{13} \bar{S}$ inhalation and extracranial recording. Clinical Science, 29 179-192.

Obrist, W. D., Thompson, H. E., King, C. H., and Wang? H. S. (1967). Determination of regional cerebral bloo $\Phi$ flow by inhalation of ${ }^{133}$ Xenon. Circulation Research, 20 124-134.

Olesen, J., Paulson, O. B., and Lassen, N. A. (1971). Regionat cerebral blood flow in man determined by the initial slope of the clearance of intra-arterially injected ${ }^{133} \mathrm{Xe}$. Stroke, 2 ? 519-540.

Paulson, O. B., Cronqvist, S., and Risberg, J. (1969). Regional cerebral blood flow: Comparison of 8-detector and 16detector instrumentation. Journal of Nuclear Medicine, 10, 164-173.

Wilkinson, I. M. S., Bull, J. W. D., Du Boulay, G. H., Marshall, J., Ross Russell, R. W., and Symon, L. (1969). Regional blood flow in the normal cerebral hemisphere. Journal Neurology, Neurosurgery, and Psychiatry, 32, 367378.

Wyper, D. J., and Rowan, J. O. (1972). An on-line system for acquisition and processing of cerebral blood flow data. Computers in Biology and Medicine, 3, 55-63.

Zierler, K. L. (1965). Equations for measuring blood flow by external monitoring of radioisotopes. Circulation Research, 16, 309-321. 